Math. Model. Nat. Phenom.

Vol. 3, No. 2, 2008, pp. 98-101

\title{
Reflections on the Origin, Meaning, and Future of Systems Biology
}

\author{
M. Santillán ${ }^{1}$ \\ Centro de Investigación y de Estudios Avanzados del IPN \\ Unidad Monterrey Vía del Conocimiento 201 \\ Parque de Investigación e Innovación Tecnológica \\ Autopista Monterrey-Aeropuerto km 9.5, 66600 Apodaca NL, México
}

The term molecular biology is so familiar nowadays that nobody questions its meaning. However, there was no consensus in the beginning about that, and even some people disputed that such a term corresponded to a new science. For instance, Kendrew wrote in a paper on the origin of molecular biology [4] that: "Biochemists point out that molecular biology is their own field, in a strict sense of the words, and sometimes deride the assumption of the phrase as a title by a group whose members were ignorant of biochemistry and indeed often hostile to it... One distinguished biochemist has referred to molecular biology as the practice of biochemistry without license."

On the other hand, molecular biologists themselves were by no means unanimous about the nature of their subject. According to Astbury [1], who introduced one of the first definitions, "molecular biology is concerned particularly with the forms of biological molecules and with the evolution, exploitation and ramification of these forms in the ascent to higher and higher levels of organization. Molecular biology is predominantly three-dimensional and structural - which does not mean, however, that it is merely a refinement of morphology. It must at the same time inquire into genesis and function." Interestingly, this definition does not even mention biological information or genetics. However, by the time the term molecular biology had become popular, in the 1950's, and many research institutes and university departments had been organized under that name, its meaning had evidently widened to include also molecular genetics [9]. And, as Kendrew points out [4], though molecular geneticists were interested in such matters as the DNA double helix, "the features of that structure that had been important to them had not been geometrical so much as topological:

\footnotetext{
${ }^{1}$ E-mail: msantillan@cinvestav.mx
} 
the one-dimensional [rather than the three-dimensional] nature of the information store and the role of the specific pairs of nitrogenous bases in replication."

Thus, As Stent remarks [9], there existed in the beginning two schools of molecular biologists: structurists and informationists, three-dimensionists and one-dimensionists, who, "although they listened politely enough to each other's seminars, had less to say to each other in terms of real intellectual communication than one might expect" [4].

The current situation of systems biology is somehow similar to that of molecular biology in its origin. Joyner and Saltin [3], for instance, claim that "before the reductionist community naively concluded they needed to reinvent and rename physiology in the guise of systems biology, investigators interested in [the physiology of] exercise were already committed to understanding the interactions of key biological responses at multiple levels of organization and integration... systems biologists have much to learn from the successes of investigators interested in exercise and even more to learn from their continuing questions." On the other hand, there is no general consensus about the meaning of systems biology:

- Some sources discuss systems biology as a field of study, particularly, the study of the interactions between the components of biological systems, and how these interactions give rise to the systems' function and behaviour (for example, the enzymes and metabolites in a metabolic pathway) [8].

- Other sources consider systems biology as a paradigm, usually defined in antithesis to the so-called reductionist paradigm. The distinction between the two paradigms is referred to in these quotations:

"The reductionist approach has successfully identified most of the components and many of the interactions but, unfortunately, offers no convincing concepts or methods to understand how system properties emerge... the pluralism of causes and effects in biological networks is better addressed by observing, through quantitative measures, multiple components simultaneously and by rigourous data integration with mathematical models" [7]

"Systems biology... is about putting together rather than taking apart, integration rather than reduction. It requires that we develop ways of thinking about integration that are as rigorous as our reductionist programmes, but different... It means changing our philosophy, in the full sense of the term" [6]

- Still other sources view systems biology in terms of the operational protocols used for performing research, namely a cycle composed of theory, computational modelling to propose specific testable hypotheses about a biological system, experimental validation, and then using the newly acquired quantitative description of cells or cell processes to refine the computational model or theory [5, 2]. 
Despite the above criticisms, which as we have seen are concomitant to a nascent field, systems biology is thriving. The number of journals advocated to this field have multiplied in the last few year, as have the number of research groups, university departments, research institutes, and published books bearing this name. The appearance of monographs on systems biology is particularly important because, in my understanding, it indicates that this field its turning mature.

It is impossible to foresee at this point how revolutionary systems biology will be, if it is going to be revolutionary at all. I personally believe that, given the advances observed in the last decade, the discovery in the near future of new fundamental laws of nature through systems biology is unlikely. If I'm correct, systems biology won't be a scientific revolution in the sense that quantum mechanics was in the early 20th century. Nevertheless, it is already changing the way biology is done. Experimental biologists are now far more receptive to mathematical modelling approaches than they were ten years ago, and this is a big success of systems biology. I envision mathematical modelling becoming a fundamental part of biological research in the short to medium terms, transforming biology into a highly predictive and quantitative science, like physics and chemistry.

Regarding the criticisms claiming that systems biology is nothing but the import of the physiology approach into cell and molecular biology, I considerer them to be mostly true. However, as we have seen with the example of molecular biology, this is how science progresses. On the one hand, the events where a new scientific discipline is created from scratch are extremely rare in the history of science. On the other hand, the necessity of young scientists to differentiate themselves from previous generations drives them to coin new names for the disciplines they carry out. From this point of view, perhaps systems biology is just a new name for an older thing, but this does not mean that systems biology does not exist. As some philosophers claim, the very act of naming something brings it into existence. Systems biology, as molecular biology before, has been born and is here to stay. How important it is meant to be? It hard to say. People have great expectations for systems biology, but only time can tell.

\section{References}

[1] W. T. Astbury. Molecular biology or ultrastructural biology? Nature, 190 (1964), 1124.

[2] C. Dollery, R. Kitney. Systems biology: a vision for engineering and medicin. Technical report, Academy of Medical Sciences and The Academy of Medical Sciences, http://www.acmedsci.ac.uk/p99puid97.html, 2007.

[3] M. J. Joyner, B. Saltin. Exercise physiology and human performance: systems biology before systems biology! J. Physiol., 586 (2008), No. 1, 9.

[4] J. C. Kendrew. How molecular biology started. Scientific American, 216 (1967), No. 3, $141-144$. 
[5] B. N. Kholodenko, F. J. Bruggeman. Mechanistic and modular approaches to modeling and inference of cellular regulatory networks. In L. Alberghina and H.V. Westerhoff, editors, Systems biology: definitions and perspectives, 143-162. Springer-Verlag, Heidelberg, 2005.

[6] D. Noble. The music of life. Oxford University Press, Oxford, 2006.

[7] U. Sauer, M. Heinemann, N. Zamboni. Getting closer to the whole picture. Science, 316 (2008), 550-551.

[8] J. L. Snoep, H.V Westerhoff. From isolation to integration, a systems biology approach for building the silicon cell. In L. Alberghina and H.V. Westerhoff, editors, Systems biology: definitions and perspectives, 13-30. Springer-Verlag, Heidelberg, 2005.

[9] G. S. Stent. That was the molecular biology that was. Science, 160 (1968), 390-395. 